\title{
Die Verschuldung der Entwicklungsländer und ihre Konsequenzen für die Vergabe von öffentlicher Entwicklungshilfe
}

\author{
Von Joachim von Stockhausen
}

"Schuldenberge belasten die Entwicklungshilfe, viele Projekte stehen mangels Mittel still«. So lautet eine Schlagzeile der deutschen Presse vom letzten Jahr. Andeutungen der Deutschen Gesellschaft für Technische Zusammenarbeit (GTZ) zufolge, müßten zahlungsunfähige Entwicklungsländer mit größeren Schwierigkeiten bei Zusagen für künftig neu beantragte Projekte rechnen; in bestimmten Ländern werde es einfach keine Neuzusagen geben.' Dabei handelt es sich ganz offensichtlich zugleich um solche Entwicklungsländer, die nach Ansicht der Weltbank jetzt und weiterhin in einem erheblichen Umfange Auslandskapital, insbesondere in Form von öffentlicher Entwicklungshilfe, benötigen:2 Die Entwicklungshilfe zu Vorzugsbedingungen müßte für Afrika im Verlauf der nächsten zehn Jahre etwa doppelt so stark steigen, wenn das Pro-Kopf-Einkommen der af rikanischen Länder mit niedrigem Einkommen jährlich um 2 Prozent und damit nur sehr bescheiden - zunehmen soll. ${ }^{3}$

Wird dem Branchenführer im Bereich des Kapitaltransfers zu Vorzugsbedingungen von der deutschen Entwicklungshilfeadministration die Gefolgschaft versagt? Im folgenden soll dem Zusammenhang von Verschuldung der Entwicklungsländer einerseits und der Vergabe öffentlicher Entwicklungshilfe andererseits näher nachgegangen werden.

\section{Das Projekt als Kern der deutschen Entwicklungshilfe}

Projekte bilden den Schwerpunkt der deutschen Zusammenarbeit mit Entwicklungsländern. Sie werden als funktional, räumlich, wirtschaftlich und zeitlich abgrenzbare Maßnahmen mit zielorientierter Aufgabenstellung definiert. ${ }^{4}$ Die Förderung von Projekten wird für einen überschaubaren, zur Erreichung eines sinnvollen Förderungszieles ausrei-

1 Schuldenberge belasten die Entwicklungshilfe. "Passauer Neue Presse“ vom 29. 8. 1984; abgedruckt in BMZ, Entwicklungspolitik - Spiegel der Presse 19/84, S. 551.

2 E. Stern, Die Entwicklungsherausforderung heute. "Finanzierung und Entwicklung" Jg. 20 (1983), Nr. 3, S. 3.

3 Weltbank, Weltentwicklungbericht 1983, S. 4. - Die Zusagen der öffentlichen Entwicklungshilfe der Bundesrepublik Deutschland für die am wenigsten entwickelten Länder (LLDC) sind in den Jahren 1982 und 1983 von 1158,9 Mio. DM auf 1248,8 Mio. DM, d. h. um 7,7\% gestiegen im Vergleich zu einem Zuwachs der gesamten Zusagen von 5,4\%. BMZ, Deutsche Entwicklungspolitik. Memorandum der Bundesregierung zur DAC-Jahresprüfung 1984/85. Bonn 1984, Tabelle 4.

4 BMZ, Leitlinien für die bilaterale Finanzielle und Technische Zusammenarbeit mit Entwicklungsländern vom 23. Februar 1984. Entwicklungspolitik - BMZ-aktuell Juni 1984, S. 4. 
chend bemessenen Zeitraum geplant und finanziert. Bei der Finanzierung wird davon ausgegangen, daß von der Bundesregierung die in ausländischer Währung anfallenden Kosten getragen und entsprechende Leistungen erbracht werden; die Finanzierung der in Landeswährung entstehenden Kosten dagegen ist vorrangig Sache des Entwicklungslandes.

Laufende Kosten für den Betrieb und die Unterhaltung des Vorhabens sind grundsätzlich vom Entwicklungsland selbst zu tragen. ${ }^{5}$ Von diesem Grundsatz kann nur in Ausnahmefällen abgewichen werden, so etwa im Falle geringer Leistungsfähigkeit des Entwicklungslandes oder des Projektträgers, insbesondere in den am wenigsten entwickelten Ländern (LLDC). In solchen Ausnahmefällen beschränkt sich die Zahlung der laufenden Kosten durch die Bundesregierung auf einen überschaubaren Zeitraum. In jedem Fall ist Voraussetzung für die Ubernahme der laufenden Kosten ein verbindlicher Plan für die von dem Entwicklungsland und der Bundesregierung zu tragenden Kostenanteile mit einer progressiven Ubernahme durch das Entwicklungsland.

Das entwicklungspolitische Anliegen einer solchen anteiligen Projektfinanzierung ist of fensichtlich. Die Regierungen der Entwicklungsländer sollen über ein finanzielles Engagement verstärkt in die Projektverantwortung einbezogen werden. Zugleich sollen sie angehalten werden, zur Entlastung ihrer Haushalte eine teilweise oder völlige Kostendeckung für die Bereitstellung öffentlicher Güter und Dienstleistungen durchzusetzen. ${ }^{6}$ Sieht man von dem fiskalpolitischen Anliegen ab - in der Praxis war es bislang nur in Ausnahmefällen im Rahmen der Projektförderung durchsetzbar - so hat die anteilige Projektfinanzierung zur Folge, daß Projekte mit relativ hohen laufenden Kosten von der deutschen Entwicklungshilfe benachteiligt werden. Dabei handelt es sich gerade um Projektansätze in den Bereichen der Landwirtschaft, des Bildungs- und Gesundheitswesens, d. h. um Bereiche, die von dem Bundesministerium für wirtschaftliche Zusammenarbeit (BMZ) als Schwerpunktbereiche der Zusammenarbeit herausgestellt werden. Die nur anteilige Projektfinanzierung übt indirekt einen Druck auf die Entwicklungsländer aus, die laufenden Ausgaben für "Altprojekte" auf ein Mindestmaß zu beschränken, um über die somit erzielte "öffentliche Ersparnis" die anteilige Finanzierung von Neuvorhaben sicherzustellen. Angesichts eines solchen initiiierten Freisetzungseffektes von Haushaltsmitteln können Klagen darüber nicht verwundern, daß beispielsweise Bildungsprojekte dadurch bedroht sind, daß der Regierung das Geld für die Bezahlung von Lehrern und sonstigen laufenden Ausgaben fehlt.

\section{Verschuldungsprobleme der Entwicklungsländer}

Nach Ansicht der Weltbank sind die Verschuldungsprobleme der meisten großen Entwicklungsländer auf einen Mangel an Liquidität und nicht auf Insolvenz zurückzufüh-

6 J. Meerman, Minimierung von Folgekosten. "Finanzierung und Entwicklung" Jg. 20 (1983), Nr. 4, S. 41 f. 
ren. ${ }^{7}$ Als ursächlich verantwortlich für den Mangel an Liquidität werden zwei miteinander verzahnte Umstände herausgestellt: (1) rückläufige Staatseinnahmen, bedingt durch die sich abschwächende Wirtschaftstätigkeit, der stagnierende Außenhandel und die Schwierigkeit, in der Rezession neue Steuern einzuführen, sowie (2) unzulängliche Leistungsfähigkeit im Sinne einer effizienten Steuerung der Ressourcenverwendung über Preise, Märkte und administrative Eingriffe sowie funktionelle Unfähigkeit, den Ertrag von Arbeit und Kapital durch eine wirksame Steuerung von Unternehmen, Projekten und Programmen sowohl im öffentlichen als auch im privaten Sektor zu maximieren. ${ }^{8}$

Die angeführten Ursachen des Schuldendienstproblemes finden u. a. ihren sichtbaren Ausdruck in der unzureichenden Bereitstellung von Mitteln, um den laufenden Betrieb und die Instandhaltung bestehender Einrichtungen zu finanzieren sowie in einer Bevorzugung von neuen Projekten. Die Weltbank hat nicht nur die Regierungen der Entwicklungsländer im Auge, wenn sie die Vernachlässigung von Wartungs- und Wiederherstellungsprogrammen zugunsten neuer Projekte kritisiert. ${ }^{9}$ In nicht wenigen Fällen werde die Leistungsfähigkeit durch das Verhalten der Entwicklungshilfe-Geber beeinträchtigt, so etwa ${ }^{10}$

- durch großzügige Bereitstellung von finanziellen Mitteln für neue Ausrüstungen, während es den Ländern überlassen bleibt, für die Beschaffung von Ersatzteilen zu sorgen;

- durch die Förderung einer unwirtschaftlichen Verbreitung unterschiedlicher Ausrüstungsarten und -modelle über liefergebundene Entwicklungshilfe oder andere $\mathrm{Be}$ schaffungsbestimmungen;

- durch die Förderung von Investitionsvorhaben, die verhindern, daß die eigenen Ressourcen des Landes in dringendere Instandhaltungsarbeiten fließen.

Nach Berechnungen der Weltbank ist von 1960-70 bis 1970-80 die Investitionsquote (in Prozent des BIP) der Entwicklungsländer von 21,3 auf 25,8 gestiegen, während zugleich der Investitionsertrag (in Prozent) von 26,8 auf 20,5 gefallen ist. Als mitverantwortlich für die gesunkenen Investitionserträge sieht die Weltbank den anhaltenden Druck an, denen sich die Regierungen der Entwicklungsländer ausgesetzt sehen, neue Vorhaben in Angriff zu nehmen. So würden häufig Investitionsprogramme beschlossen, die ihre finanziellen Möglichkeiten und Verwaltungskapazitäten überfordern. Die Folgen seien Verzögerungen bei der Projektverwirklichung sowie daraus sich ergebende Produktionseinbußen. Unter der Annahme von Opportunitätskosten in Höhe von 10 Prozent für den Kapitaleinsatz hat die Weltbank für eine Gruppe von Entwicklungsländern die Kosten

7 Weltbank, a.a.O. 1983, S. 4.

Weltbank, a.a.O. 1983, S. 50.

Weltbank, a.a.O. 1983, S. 108

10 Weltbank, a.a.O. 1983, S. 54. - Das BMZ hat sich of fensichtlich die Kritik der Weltbank zu eigen gemacht, wenn es jüngst betont, daß bei der Entscheidung über die Verwendung neuer Zusagen der Gesichtspunkt im Vordergrund stehe, zunächst bestehende Kapazitäten zu verbessern und zu erhalten, ehe neue Vorhaben begonnen werden. BMZ, Deutsche Entwicklungspolitik a.a.O. 1984, S. 22. 
einer zweijährigen Verzögerung in der Projektdurchführung auf 20 Prozent der Investitionskosten geschätzt. ${ }^{11}$

Eine Erhöhung der Leistungsfähigkeit allein im mikroökonomischen Projektbereich reicht jedoch nicht aus bzw. ist sogar ohne Einbezug des makroökonomischen Bereichs nicht möglich, wobei es sich insbesondere um die Zusammenhänge zwischen Preisverzerrungen und Wirtschaftswachstum handelt. Statistische Analysen dieses Zusammenhanges machen deutlich, daß die durchschnittliche Wachstumsrate der Länder mit geringen Verzerrungen in den siebziger Jahren etwa 7 Prozent pro Jahr und damit 2 Prozentpunkte mehr als der Durchschnitt aller Länder erreichte. Demgegenüber lag das durchschnittliche Wachstum der Länder mit starken Verzerrungen bei etwa 3 Prozent pro Jahr oder 2 Prozentpunkte unter dem Gesamtdurchschnitt. ${ }^{12}$

Starke Preisverzerrungen sind mit niedrigen inländischen Ersparnissen und mit einer geringen Wertschöpfung je Investitionseinheit verbunden. Die Bedeutung dieser Preisverzerrungen für das Wirtschaftswachstum und die Schuldendienstfähigkeit verdeutlicht Ubersicht 1 am Beispiel von verschiedenen asiatischen Ländergruppen. Der ausgewiesene "kritische Zinssatz" gibt die höchste Zinssatzbelastung an, die ein Land für ausländisches Fremdkapital tragen kann, wenn die Zinsbelastung die Produktivitätskapazität nicht übersteigen soll. ${ }^{13}$ In allen drei Ländergruppen ist im Zeitverlauf der marginale Kapitalkoeffizient gestiegen, während, mit Ausnahme der Schwellenländer, die marginale Sparquote gesunken ist. Damit einhergegangen ist eine Abnahme der Wachstumsrate des BSP und der Schuldendienstfähigkeit.

Sensitivitätsanalysen machen deutlich, daß eine Erhöhung der marginalen Sparquote den stärksten Einfluß auf eine Erhöhung der Schuldendienstfähigkeit hat, gefolgt von einer Abnahme des marginalen Kapitalkoeffizienten und einer Zunahme der Wachstumsrate des $\mathrm{BSP}$.

Der starke Einfluß der Sparquote auf die Schuldendienstfähigkeit der Entwicklungsländer hat weitreichende Konsequenzen für die Entwicklungshilf epolitik; denn empirische Untersuchungen haben überwiegend einen Verdrängungseffekt von inländischen Ersparnissen durch Kapitalhilfe nachgewiesen. Danach können ausländische Kapitalzuflüsse im allgemeinen nicht als vollwertiges Substitut für die inländische Mobilisierung von Ersparnissen angesehen werden. Nach Fischer spricht vieles für die These, daß je entwikkelter und funktionsfähiger der Finanzsektor in Entwicklungsländern ist, um so weniger wird die inländische Sparquote von (privaten) Kapitalzuflüssen aus dem Ausland berührt. ${ }^{14}$

11 Weltbank, a.a.O. 1983, S. 53.

12 Weltbank, a.a.O. 1983, S. 75.

13 J. Lee, The External Debt-Servicing Capacity of Asian Developing Countries. "Asian Development Review" (1983), No. 2, S. 78.

14 B. Fischer, Liberalisierung der Finanzmärkte und wirtschaftliches Wachstum in Ent wicklungsländern. Kieler Studien Nr. 172. Tübingen 1982, S. $77 \mathrm{ff}$. 


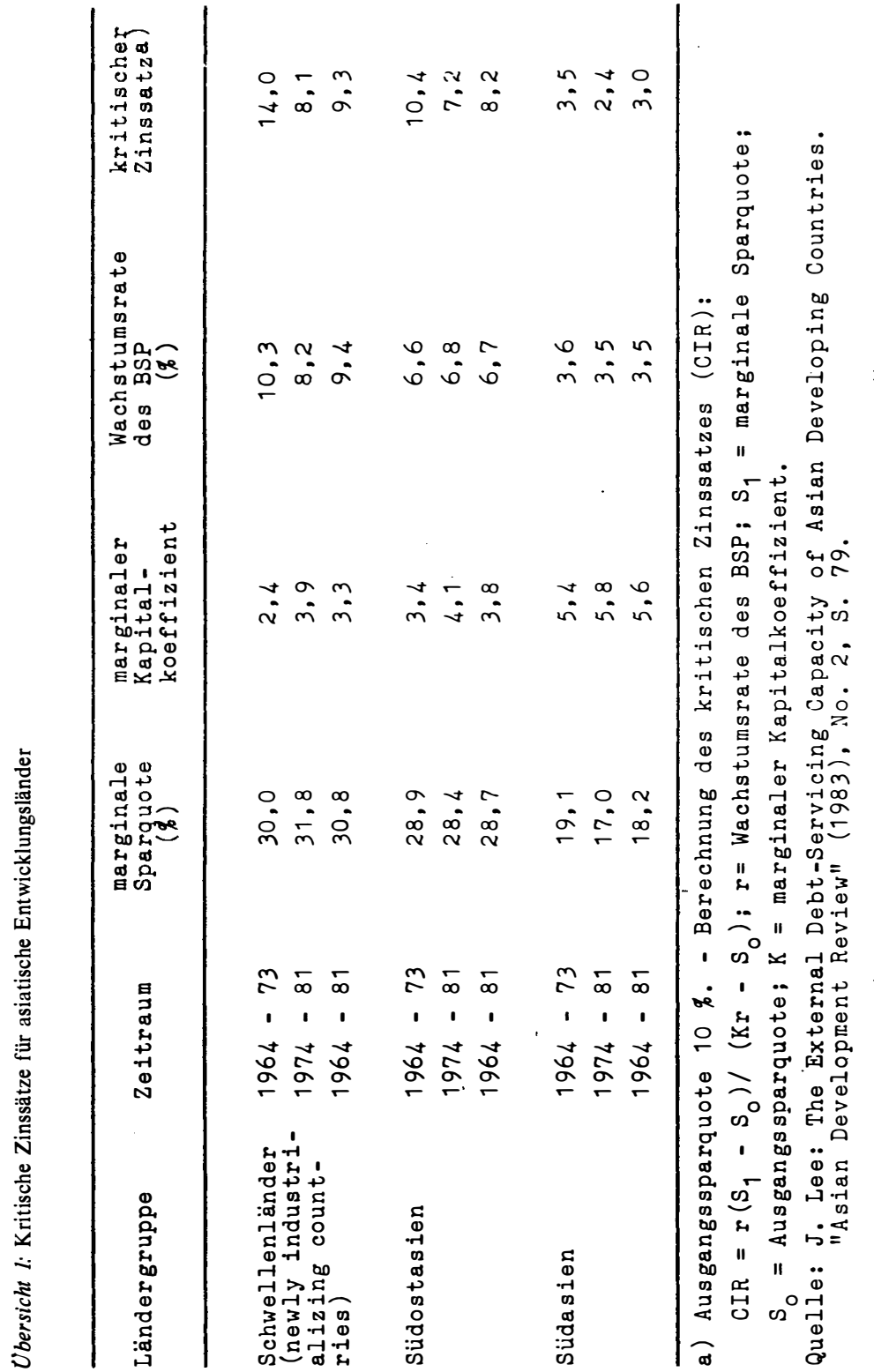


Angesichts dieser Beziehungen zwischen Kapitalhilfe, inländischer Sparquote und Schuldendienstfähigkeit ist es überraschend, wenn auch im Sinne der Projekthilfe konsequent, daß die Refinanzierung von Entwicklungsbanken, Kreditgenossenschaften und ähnlichen Instituten mit Mitteln der Entwicklungshilfe vorwiegend als Instrument zur Kreditfinanzierung von kleinen und mittleren Betrieben und weniger als Instrument zur Entwicklung des Finanzsektors angesehen wird. ${ }^{15}$ Erst in jüngerer Zeit wird eine geänderte Sichtweise dadurch deutlich, daß das BMZ ein Forschungsvorhaben vergeben hat, das sich mit den Ansatzmöglichkeiten für die Mobilisierung von Sparkapital zur Entwicklungsfinanzierung befaßt.

\section{Flexible Entwicklungshilfe der Weltbank}

Auf die Zunahme der Zahlungsbilanzdefizite der Entwicklungsländer hat die Weltbank Anfang 1983 mit ihrem Sonderhilfeprogramm flexibel reagiert. Sein Ziel ist es, die Entwicklungsländer dabei zu unterstützen, ihre Entwicklungsbemühungen trotz nachteiliger äußerer Umstände wieder aufzunehmen. Eine Komponente dieses Programms ist die Vergabe von Strukturanpassungskrediten. Voraussetzung für ihre Vergabe ist, daß ein Land bereit ist, seine Politik und seine Programme so zu ändern, daß sich die Volkswirtschaft in einer angemessenen Frist an die veränderte Lage der Weltwirtschaft anpassen kann, ohne die langfristigen Wachstumsziele zu gefährden. Dieses bedeutet, daß das Leistungsbilanzdefizit auf ein Niveau reduziert werden soll, das mit der Höhe an Auslandskapital im Einklang steht, die das Land auch zu normalen Bedingungen hätte aufnehmen können, ohne seine Schuldendienstkapazität überzubeanspruchen. ${ }^{16}$ Im wesentlichen geht es darum, die Effizienz von Landwirtschaft und Industrie zu verbessern, die Exporte hinsichtlich Höhe und Struktur auf der Grundlage komparativer Vorteile zu fördern, inländische Energiequellen zu entwickeln und effizienter zu nutzen, den Konsum zu beschränken und die Sparquote zu erhöhen.

Eine weitere Komponente des Sonderhilfsprogramms ist die zusätzliche Finanzierung laufender Projekte. Dabei läßt sich die Weltbank von der Uberlegung leiten, daß es in diesem Stadium sinnvoller ist, Ländern zu helfen, Entwicklungsprojekte mit hoher Priorität zu vollenden und vorhandene Kapazitäten auszunutzen, als Geld in neue Investitionen fließen zu lassen. ${ }^{17}$

Im allgemeinen finanziert die Weltbank, ähnlich wie die Bundesregierung, lediglich die Devisenkosten von Projekten. Bei Ländern mit mittleren und höheren Einkommen allerdings können Ausnahmen von dieser Regel gemacht werden, wenn die Devisenkompo-

15 Vgl. J. von Stockhausen, Staatliche Agrarkreditpolitik und ländliche Finanzmärkte in den Ländern der Dritten Welt. Untersuchungen über das Spar-, Giro- und Kreditwesen, Abteilung Wirtschaftswissenschaft Bd. 118. Berlin 1984.

16 E. P. Wright, Weltbankkredite zur Strukturanpassung. "Finanzierung und Entwicklung" Jg. 17 (1980), Nr. 3, S. 21.

17 E. Stern, a.a.O. 1983, S. 4. 
nente sehr gering ist. In diesen Fällen besteht die Möglichkeit, bis zu 35 Prozent der gesamten Kosten zu finanzieren. In den ärmsten Ländern finanziert die Weltbank bis zu 95 Prozent der Projektkosten.

Mit dem Sonderhilfsprogramm ist die Weltbank in die Lage versetzt worden, ihre üblichen Kostenübernahmekriterien auf einer länder- und projektspezifischen Grundlage zu verändern, und zwar a) durch die Reduzierung des Projektumfanges bei Einhaltung des gesamten Betrages an Bankmitteln, b) durch die Gewährung von Ergänzungskrediten, einschließlich rückwirkender Darlehen und c) durch die verbesserte Ausstattung der Darlehen, um so die Laufzeit der Projekte zu vermindern. ${ }^{18}$

Ein weiteres Anliegen dieses Programmes besteht darin, andere Kreditgeber aufzufordern, ähnliche Anstrengungen zu unternehmen und Maßnahmen zu erwägen. Damit ist direkt die Bundesregierung mit der Ausgestaltung ihrer Entwicklungshilfepolitik angesprochen.

\section{Entwicklungshilf epolitik im Rahmen deutscher Gesamtpolitik}

Grundlage der deutschen Entwicklungshilfe ist ein partnerschaftlicher Dialog mit den Entwicklungsländern über allgemeine und aktuelle Fragen der Zusammenarbeit. Der Dialog soll im Sinne eines Interessenausgleiches geführt werden, bei dem die beiderseitigen Anliegen vorgetragen und besprochen werden. Dabei hält es die Bundesregierung für erforderlich, daß das Entwicklungsland durch entsprechende Eigenanstrengungen geeignete strukturelle Voraussetzungen und Rahmenbedingungen für die von der Bundesregierung geförderten Vorhaben und für die gesamte Entwicklung schafft. ${ }^{19}$ Als erforderliche Rahmenbedingungen bzw. Handlungen führt das BMZ explizit auf, ${ }^{20}$

- daß im Rahmen der entwicklungspolitisch sinnvollen Verwendung der Mittel bei der Vergabe von Lieferungen und Leistungen die Beschäftigungswirksamkeit in der Bundesrepublik Deutschland zu berücksichtigen ist und

- daß die Strukturen innerhalb des Projektes und in seinem Umfeld derartig beschaffen sind bzw. geschaffen werden, daß der Erfolg der Förderung sichergestellt ist; die angesprochenen Strukturen sollen im Einklang mit entsprechenden Vorhaben anderer bilateraler und multilateraler Geber stehen, soweit dieses für die Zielerreichung des Vorhabens notwendig ist.

Der solchermaßen angestrebte Politikdialog umfaßt damit drei Ebenen: die Interessenlage der Bevölkerung der Bundesrepublik, die der Empfängerländer und die der anderen Geber von Entwicklungshilfe.

Nach dem Regierungswechsel in Bonn im Jahre 1982 ist als eine der vordringlichsten Aufgaben des neuen Bundesministers für wirtschaftliche Zusammenarbeit herausgestellt

18 Weltbank, Jahresbericht 1983, S. 42.

19 BMZ, a.a.O. 1984, S. 10.

20 BMZ, a.a.O. 1984, S. 12 f. 
worden, der Entwicklungshilfepolitik wieder die ihr verlorengegangene Bedeutung innerhalb der Gesamtpolitik der Bundesregierung zurückzugeben. Als wichtiges Instrument der westdeutschen Politik müsse die Entwicklungshilfe in Úbereinstimmung mit den außenpolitischen Zielen geleistet werden. Zuständig für die außenpolitischen Beziehungen sei das Auswärtige Amt, so daß es keine eigenständige Außenpolitik des BMZ geben könne. ${ }^{21}$

Entwicklungshilfepolitik als Teil der Außenpolitik zu betrachten, stellt sie in den Kontext gesamtgesellschaftlicher Systemwahrung, mit anderen Worten, Entwicklungshilfepolitik wirkt in dem Umfange dysfunktional, wie sie nicht darauf ausgerichtet ist, die internationale Umwelt in der Weise zu beeinflussen, daß die Bestandserhaltung des bundesrepublikanischen Gesellschaftssystems gewahrt, möglichst gefördert wird. Regional auf die Entwicklungsländer ausgerichtet und versehen mit den Instrumenten der Kapitalhilfe und Technischen Hilfe fungiert die Entwicklungshilfepolitik parallel und gleichzeitig mit anderen Teilpolitiken wie Diplomatie, Außenhandelspolitik usw. Aus diesem funktionalen Verständnis folgt, daß Entwicklungshilfepolitik in der Tat nicht Politik sui generis sein kann; als gesonderte Form der Außenpolitik unterscheidet sie sich jedoch von anderen Teilpolitiken. ${ }^{22}$

Während es das Anliegen der Außenhandelspolitik ist, unter Wahrung der bestehenden internationalen Gesellschaftsstrukturen die ökonomische Verflechtung der Bundesrepublik Deutschland mit der übrigen Welt zu fördern, gibt sich die Entwicklungshilfepolitik mit den bestehenden Strukturen nicht zufrieden. Sie bemüht sich darum, das Niveau der Integration der Entwicklungsländer in den Weltmarkt zu verbessern. ${ }^{23}$ Aus diesem spezifischen Bezug der Entwicklungshilfepolitik folgt, daß es zu ihr kein funktionales Äquivalent gibt. Hieraus folgt aber auch, daß Entwicklungshilfepolitik in dem Maße scheitern muß, wie das genuine entwicklungshilfepolitische Rationalitätskalkül durch die Rationalitätskalküle anderer Teilpolitiken der Außenpolitik, wie z. B. der Außenwirtschaftspolitik, dominiert wird. Beispiel hierfür ist eine dominierende Begründung der Entwicklungshilfe mit kurzfristigen Beschäftigungswirkungen und dem Absatz von landwirtschaftlichen Uberschüssen. Denn eine Entwicklungshilfepolitik, die sich solchen fadenscheinigen ökonomischen Begründungen beugt, ist der Gefahr ausgesetzt, daß sie ihrer eigentlichen außenwirtschaftlichen Begründbarkeit, nämlich der Expansion von Absatzmärkten der Zukunft über eine Erhöhung der Massenkaufkraft in den Entwicklungsländern, zuwider handelt. ${ }^{24}$

21 K. Nathorp, Entwicklungspolitik in Routine erstarrt. "Frankfurter Allgemeine Zeitung" vom 9. 10. 1982; abgedruckt in BMZ, Entwicklungspolitik - Spiegel der Presse 21/82, S. 634.

22 M. Glagow, Wider das ökonomische Mißverständnis - zur Funktion von Entwicklungspolitik. In: M. Glagow (Hrsg.): Deutsche Entwicklungspolitik: Aspekte und Probleme ihrer Entscheidungsstruktur. Bielef elder Studien zur Entwicklungssoziologie Bd. 19. Saarbrücken - Fort Lauderdale 1983, S. $30 \mathrm{ff}$.

23 U. Schimank, Das außenpolitische Interorganisationsnetz als Hemmnis einer eigenständigen deutschen Entwicklungspolitik: Heteronomie und Autonomisierungsbestrebungen des Bundesministeriums für wirtschaftliche Zusammenarbeit. In: M. Glagow (Hrsg.), a.a.O. 1983, S. $61 \mathrm{ff}$.

24 Vgl. H. Elsenhans, Nord-Süd-Beziehungen. Geschichte - Politik - Wirtschaft. Urban-Taschenbücher Politik Bd. 365. Stuttgart 1984. 
Politikdialog in der Entwicklungshilfe sollte damit zunächst nach innen orientiert sein, um die unterschiedlichen Bezugsfelder von Außenpolitik, Außenwirtschaftspolitik und Entwicklungshilfepolitik deutlich zu machen. Dabei gilt es auch, dem Steuerzahler das Spannungsfeld zwischen gesellschaftsreformerischer Entwicklungshilfe einerseits und einer vordergründigen beschäftigungswirksamen staatlichen Entwicklungshilfe andererseits aufzuzeigen. Auf dieses Spannungsfeld hat jüngst Dr. Volkmar Köhler, Parlamentarischer Staatssekretär beim BMZ, indirekt hingewiesen: "Wenn wir in einer Zeit, in der die 'Kassen ' leer sind, die dringend erforderliche Strukturanpassung in den Entwicklungsländern fördern wollen, dann müssen wir unsere eigenen nationalen Interessen und möglicherweise auch Egoismen überwinden und zu einer wirksamen Geberkoordination finden “. ${ }^{25}$ Doch ist die deutsche Bevölkerung zu solchen Opfern bereit?

\section{Einstellung der deutschen Bevölkerung zur Entwicklungshilfe}

Staatliche Entwicklungshilfe wird von dem Steuerzahler getragen. Sie setzt demzufolge sein grundsätzliches Einverständnis voraus. Wie aber schätzt der deutsche Steuerzahler den Gedanken der Entwicklungshilfe und die praktische Ausgestaltung der staatlichen Entwicklungshilfepolitik ein?

Die allgemeine Zustimmung der deutschen Wahlbevölkerung zu dem Gedanken der Entwicklungshilfe zeigt in den letzten sechs Jahren eine steigende Tendenz (vgl. Úbersicht 2).

Ubersicht 2: Einstellung zur Entwicklungshilfe

\begin{tabular}{lllll}
\hline & Nov. & Okt. & Okt. & Nov. \\
& 1977 & 1979 & 1981 & 1983 \\
\hline $\begin{array}{l}\text { eher dafür } \\
\text { eher dagegen }\end{array}$ & $62 \%$ & $71 \%$ & $67 \%$ & $74 \%$ \\
$\begin{array}{l}\text { weiß nicht/ } \\
\text { keine Angabe }\end{array}$ & $23 \%$ & $18 \%$ & $20 \%$ & $15 \%$ \\
\hline & $16 \%$ & $11 \%$ & $13 \%$ & $11 \%$ \\
\hline
\end{tabular}

Quelle: Infratest Sozialforschung, Einstellung der Bevölkerung zur Entwicklungshilfe und Entwicklungspolitik 1983. Kommentierung der wichtigsten Ergebnisse. München 1984, S. 7.

25 V. Köhler, Zielvorstellungen und Maßnahmen zur ländlichen Entwicklung in der Dritten Welt. Rede während der Fachtagung an der Universität Hohenheim "Ländliche Entwicklung in der Dritten Welt" am 12. Dezember 1983. 
Mit dieser zunehmend positiven Einstellung konnte die praktische Entwicklungshilfe der Bundesregierung nicht Schritt halten. Weitgehend unverändert äußert sich knapp die Hälfte der Befragten positiv, etwas mehr als ein Viertel steht ihr skeptisch gegenüber und ein weiteres Viertel vermag die Arbeit der Bundesregierung nicht zu beurteilen (vgl. Ubersicht 3).

Ubersicht 3: Einstellung zur Entwicklungshilfe der Bundesregierung

\begin{tabular}{lllll}
\hline & Nov. & Okt. & Okt. & Nov. \\
& 1977 & 1979 & 1981 & 1983 \\
\hline $\begin{array}{l}\text { eher positiv } \\
\text { eher negativ }\end{array}$ & $46 \%$ & $53 \%$ & $47 \%$ & $48 \%$ \\
weiß nicht/ & $27 \%$ & $25 \%$ & $29 \%$ & $27 \%$ \\
keine Angabe & $27 \%$ & $22 \%$ & $24 \%$ & $24 \%$ \\
\hline
\end{tabular}

Quelle: Infratest Sozialforschung, a.a.O. 1984, S. 8.

Wie ist die unterschiedliche Einschätzung des Gedankens und der praktischen Politik der Entwicklungshilfe zu erklären? Sicherlich spielt hier ein Informationsdefizit eine Rolle, denn nahezu konstant über die Jahre haben nur 12 Prozent der Bevölkerung Informationsmaterial von dem BMZ erhalten. ${ }^{26}$ Und wohl nicht zuletzt aus diesem Grunde stellt das BMZ die Bedeutung der nichtstaatlichen Entwicklungsträger, und hier vor allem die der Kirchen, gerade auf dem Gebiet der entwicklungspolitischen Bildungs- und Offentlichkeitsarbeit besonders heraus: Anhand von anschaulichen Beispielen vermitteln die nichtstaatlichen Träger Verștändnis für die Probleme in den Entwicklungsländern sowie Einblicke in die sozialen und wirtschaftlichen Zusammenhänge des Entwicklungsprozesses. Sie stärken somit die gesellschaftliche Verantwortung in der Bundesrepublik Deutschland für eine Verbesserung der Verhältnisse in den Entwicklungsländern. ${ }^{27}$ Der Offentlichkeitsarbeit ist es wohl gelungen, die Einstellung der Bevölkerung zur Entwicklungshilfe positiv zu beeinflussen, nicht aber ihre praktische Umsetzung durch die Bundesregierung in ein günstiges Licht zu rücken. Dieses gilt insbesondere für die von Infratest so bezeichneten wengagierten bedingungslosen Befürworter" - ca. 11 Prozent der Wahlbevölkerung im Jahre 1983. ${ }^{28}$ Von allen Befürwortern haben sie das größte ent-

26 Inf ratest Sozialforschung, Einstellung der Bevölkerung zur Entwicklungshilfe und Entwicklungspolitik 1983. Materialband. München 1984, S. 59.

27 BMZ, Journalistenhandbuch Entwicklungspolitik 1984. Bonn 1984, S. 184.

28 Infratest Sozialforschung, Einstellung der Bevölkerung zur Entwicklungshilfe und Entwicklungspolitik 1983. Kommentierung der wichtigsten Ergebnisse. München 1984, S. 19. 
wicklungspolitische Interesse, zugleich bejahen sie am wenigsten die Arbeit der Bundesregierung in diesem Bereich. Bei diesen Befürwortern ist die jüngere Generation sehr stark repräsentiert, mehr als 40 Prozent der Befragten haben eine höhere Schulbildung und 28 Prozent dieses Personenkreises würde bei einer Bundestagswahl "am nächsten Sonntag" den Grünen ihre Stimme geben.

Ein differenzierteres Bild über die Beurteilung der von der Bundesregierung geleisteten Entwicklungshilfe durch die Wahlbevölkerung ergibt sich dann, wenn die Befragten nach Berufsgruppen unterschieden werden (vgl. Ubersicht 4).

Ubersicht 4: "Eher positive" Beurteilung der von der Bundesregierung geleisteten Entwicklungshilfe (in \%)

\begin{tabular}{|c|c|c|c|c|}
\hline $\begin{array}{l}\text { Beruf des } \\
\text { Haushaltsvorstandes }\end{array}$ & $\begin{array}{l}\text { Nov. } \\
1977\end{array}$ & $\begin{array}{l}\text { Okt. } \\
1979\end{array}$ & $\begin{array}{l}\text { Okt. } \\
1981\end{array}$ & $\begin{array}{l}\text { Nov. } \\
1983\end{array}$ \\
\hline Arbeiter (un-/angelernt) & 39 & 35 & 44 & 48 \\
\hline $\begin{array}{l}\text { Facharbeiter } \\
\text { Beamte }\end{array}$ & 45 & 48 & 42 & 40 \\
\hline $\begin{array}{l}\text { (einf./mittl./gehob. Dienst) } \\
\text { Angestellte }\end{array}$ & 56 & 72 & 53 & 62 \\
\hline $\begin{array}{l}\text { (ausführende/qualifizierte) } \\
\text { Leitende Beamte/ }\end{array}$ & 52 & 61 & 50 & 52 \\
\hline $\begin{array}{l}\text { Angestellte } \\
\text { Selbständige }\end{array}$ & 54 & 63 & 60 & 49 \\
\hline (mittl./größere/freiberufl.) & 32 & 68 & 58 & 49 \\
\hline Selbständige (kleinere) & 41 & 43 & 40 & 59 \\
\hline Landwirte & 20 & 44 & 35 & 46 \\
\hline
\end{tabular}

Quelle: Infratest Sozialforschung, Einstellung der Bevölkerung zur Entwicklungshilfe und Entwicklungspolitik 1983. Materialband. München 1984, S. 12.

Mit allen Vorbehalten lassen sich grob drei Gruppen unterscheiden: mit steigender, abnehmender und stagnierender Tendenz einer "eher positiven" Beurteilung der von der Bundesregierung geleisteten Entwicklungshilfe.

Zur Gruppe mit steigender Tendenz gehören un- und angelernte Arbeiter, kleinere Selbständige und Landwirte.

Die steigende Zustimmung der un- und angelernten Arbeiter zur Entwicklungshilfe der Bundesregierung dürfte $u$. a. auf die von Entwicklungshilfeminister Dr. Warnke öffentlich betonte und von seinem Vorgänger bereits praktizierte Beschäftigungswirksamkeit der deutschen Entwicklungshilfe zurückzuführen sein. Durch zahlreiche Interviews in der Tagespresse dürfte es ihm gelungen sein, diesen Aspekt den Arbeitern nahegebracht zu haben. Diese Sozialgruppe, die von der Beschäftigungslosigkeit in der Bundesrepublik besonders betroffen ist, bleibt sicher nicht unberührt, wenn ihr gesagt wird, daß z. Z. 
125000 Beschäftigte an der Verwirklichung von Entwicklungshilfeprojekten in der Dritten Welt arbeiten und die Entwicklungshilfe somit im Einklang mit beschäftigungspolitischen Zielen steht. ${ }^{29}$

Die sprunghafte Zustimmung der kleineren Selbständigen dürfte mit der von Entwicklungshilfeminister Dr. Warnke besonders herausgestellten Kleingewerbe- und Handwerksförderung in Zusammenhang gebracht werden können, für die das BMZ ein umfangreiches Instrumentarium entwickelt hat. ${ }^{30}$

Befragungen von Landwirten über ihre Motive für die Vergabe von staatlicher Entwicklungshilfe haben eine Dominanz von wirtschaftlichen Motiven zutage treten lassen, wobei die Erschließung von Absatzmärkten für landwirtschaftliche Uberschüsse und Garantien für Rohstofflieferungen besonderes Gewicht haben. ${ }^{31}$ Einbezogen in die Schaffung von Absatzmärkten ist insbesondere die Nahrungsmittelhilfe der Bundesregierung, die im Jahre 1981 einen Anteil an der öffentlichen Entwicklungshilfe von 8,7 \% hatte. ${ }^{32}$ Darüber hinaus ist die EG nach der USA der zweitgrößte Nahrungsmittelhilfe-Geber. Ihre Nahrungsmittelhilfe macht mehr als $30 \%$ der gesamten gemeinschaftlichen Entwicklungshilfe aus; sie ist damit zugleich auch das wichtigste weltweite Entwicklungsinstrument der EG. ${ }^{33}$

Zu der Gruppe mit tendenziell abnehmender Zustimmung können die leitenden Beamten und Angestellten, mittlere, größere und freiberufliche Selbständige sowie die Facharbeiter gerechnet werden.

Leitende Beamte und Angestellte lassen sich nur schwer einer eindeutigen Interessenbzw. Einstellungslage zur Entwicklungshilfe zuordnen. Für ihre Einstellung ist charakteristisch, daß sie die Aufgabe der Industrienationen, den Entwicklungsländern zu helfen, grundsätzlich bejahen; sie möchten die Vergabe von Entwicklungshilfe an möglichst wenige Vorbedingungen knüpfen, so etwa daran, daß sich das Empfängerland für die westliche Demokratie entscheidet. Ihre abnehmend positive Beurteilung der von der Bundesregierung geleisteten Entwicklungshilfe steht möglicherweise in Verbindung mit der an Gewicht gewonnenen, eigeninteressenorientierten politischen und wirtschaftlichen Konditionierung der deutschen Entwicklungshilfe. Ganz allgemein hat in der Bevölkerung das politische Motiv, Entwicklungshilfe als Maßnahme gegen den Kommunismus zu gewähren, seit den 60er Jahren deutlich abgenommen; z. Z. wird es nur von einer sehr kleinen Bevölkerungsgruppe vertreten. ${ }^{34}$

Die abnehmende Tendenz bei den mittleren und größeren Selbständigen dürfte mit der seit Jahren geführten kritischen Diskussion über das Entwicklungsländer-Steuergesetz

29 BMZ, Journalistenhandbuch . . a.a.O. 1984, S. 196 f.

30 BMZ, Deutsche Unternehmer und Entwicklungsländer. Bonn 1982.

31 J. von Stockhausen, Staat und landwirtschaftliche Entwicklung in den Ländern der Dritten Welt. "Berichte über Landwirtschaft “, Bd. 63 (1985), Nr. 1, S. 140 f.

32 BMZ, Fünfter Bericht zur Entwicklungspolitik der Bundesregierung. Deutscher Bundestag - 9. Wahlperiode, Drucksache 9/2411. Bonn 1983, S. 161.

33 BMZ, Journalistenhandbuch ... a.a.O. 1984, S. 134.

34 S. Klengel, Entwicklungshilfe und öffentliche Meinung (1959-1983). Bericht der Deutschen Stiftung für internationale Entwicklung (DOK 1194C). Bonn 1983, S. 11. 
und seine Aufhebung zum 31. Dezember 1981 in Verbindung stehen. Dieses Gesetz diente dazu, steuerliche Hemmnisse und Nachteile bei Auslandsinvestitionen abzubauen. Besondere Schwierigkeiten bereitet die Erklärung der abnehmenden Zustimmung der Facharbeiter. Ihre altruistische Haltung zur Entwicklungshilfe liegt am unteren Ende der Skala der in Úbersicht 4 aufgeführten Berufsgruppen. ${ }^{35}$ Demzufolge dürften wohl die Ursachen bei ihrer eigennützigen Motivlage zu suchen sein. Untersuchungen zeigen, daß sich bei einer gleich großen Ausweitung der Importe und Exporte mit den Entwicklungsländern die saldierten Freisetzungseffekte von Arbeitsplätzen auf unausgebildete Arbeitskräfte konzentrieren, während ausgebildete Arbeitskräfte verstärkt benötigt werden; und dieses trifft um so mehr zu, je höher das Ausbildungsniveau ist. ${ }^{36}$ Dem BMZ ist es offensichtlich nicht gelungen, die Beschäftigungswirksamkeit der von ihm verfolgten Entwicklungshilfepolitik gerade für die Facharbeiter überzeugend deutlich zu machen mit der Folge einer zunehmend reservierten Einstellung zur öf fentlichen Entwicklungshilfe.

Bei der dritten Gruppe, nämlich den Beamten des einfachen bis gehobenen Dienstes und den ausführenden und qualifizierten Angestellten handelt es sich um eine Sozialgruppe, die ein überdurchschnittliches Interesse an der Entwicklungshilfe hat und die nicht ideologisch fixiert ist, so daß es für sie auch beispielsweise keine Rolle spielt, ob das Entwicklungsland kommunistisch orientiert ist oder nicht. ${ }^{37} \mathrm{Ihr}$ ausgeprägtes Interesse ist mit einer altruistischen Einstellung zur Vergabe von Entwicklungshilfe verbunden. Schwankungen in der positiven Beurteilung der staatlichen Entwicklungshilfe, wie sie in Ubersicht 4 zum Ausdruck kommen, dürften das Ergebnis einer kritischen Auseinandersetzung mit tagespolitischen Entscheidungen über ihre Ausgestaltung sein.

\section{Freiheitsgrad der deutschen Entwicklungshilf epolitik}

Angesichts der skizzierten Einstellung der deutschen Wahlbevölkerung zur Vergabe von Entwicklungshilfe ganz allgemein und zu ihrer Ausgestaltung durch das BMZ insbesondere ist die Frage zu stellen, über welchen politischen Freiheitsraum die deutsche Entwicklungshilfeadministration verfügt. Ist die deutsche Bevölkerung zu den von Köhler angesprochenen Opfern bereit, wie sie beispielsweise zu erbringen sind, wenn mit einer verstärkten Hinwendung zur Befriedigung der menschlichen Grundbedürfnisse in den Entwicklungsländern wirklich ernst gemacht würde? Eine solche Ausrichtung hätte nämlich u. a. zur Folge, daß in einem verstärkten Maße exportunwirksame, laufende Projektausgaben bestritten werden müßten und demzufolge auf einen Teil jener Rückwirkungen zu verzichten wäre, der sich bei einer Beibehaltung der bisherigen Vergabe-

35 Inf ratest Sozialforschung, Einstellung ... Materialband a.a.O. 1984, S. 23.

36 D. Schumacher, Arbeitsteilung mit Entwicklungsländern und Strukturwandel in der Bundesrepublik Deutschland. "Konjunkturpolitik" Jg. 28 (1982), S. 313 f.

37 Infratest Sozialforschung, Einstellung ... Kommentierung a.a.O. 1984, S. 17 f. 
struktur erzielen ließe. ${ }^{38}$ Oder wie steht es um den Bundestagsbeschluß vom März 1982, demzufolge bei der Vergabe von Entwicklungshilfe künftig berücksichtigt werden soll, wieviel das einzelne Empfängerland für Waffenkäufe aufwendet? Wie beurteilt beispielsweise der deutsche Bürger die auf dem Bundestagsbeschluß beruhende Entscheidung des Haushaltsausschusses des Bundestages Ende 1982, die Entwicklungshilfe für Indien um 10 Millionen DM zu verkürzen, weil die Regierung in Neu Dehli zuviel Geld für die Rüstung ausgäbe und dadurch die wirtschaftliche und soziale Entwicklung des Landes verzögere, auch wenn dadurch möglicherweise deutsche Exportmöglichkeiten gefährdet werden? ${ }^{39}$

Der dem BMZ gegebene innenpolitische Spielraum zur Ausgestaltung der Entwicklungshilfepolitik kann durch die Einstellung der Wahlbevölkerung zu dem allgemeinen Gedanken der Entwicklungshilfe einerseits und durch ihre Einstellung zur praktischen Ausgestaltung durch die Bundesregierung andererseits markiert werden. Ende 1983 stimmten $74 \%$ der Bevölkerung dem Entwicklungshilfegedanken zu, doch nur $48 \%$ hatten eine eher positive Einstellung zur Entwicklungshilfe der Bundesregierung. Dabei ist zugleich zu berücksichtigen, daß die Spendenfreudigkeit der deutschen Bevölkerung im Steigen begriffen ist. Mit rd. 114 Mio. DM erreichte beispielsweise Misereor im Jahre 1983 das bislang höchste Spendenaufkommen.

Man muß wohl davon ausgehen, daß sich altruistische und eigennützige Motive zur Vergabe von Entwicklungshilfe keineswegs ausschließen. Dieses machten auch die von Infratest unterschiedenen Einstellungsdimensionen deutlich:40 Die höchste Zustimmung mit über $80 \%$ erhielten moralische Motive wie Hungernde zu unterstützen, Solidarität mit Entwicklungsländern zu pflegen, Hilfe von außen zu gewähren, damit die Entwicklungsländer aus ihrem Elend herauskommen, wobei die Entwicklungsgelder einer breiten Bevölkerung zugute kommen und richtig eingesetzt werden müssen. Diese Einstellungsdimensionen sind gekoppelt mit einer vergleichsweise geringen, wenn auch steigenden Bereitschaft, Entwicklungshilfe auch dann zu gewähren, wenn sich daraus für eine gewisse Zeit wirtschaftliche Nachteile ergeben.

Die staatliche Entwicklungshilfeadministration gerät in die Gefahr, ihren politischen Freiheitsraum zu beschneiden, wenn sie das genuine entwicklungspolitische Rationalitätskalkül, wie es durch die Einstellungsdimensionen der Bevölkerung zum Ausdruck kommt, durch die Rationalitätskalküle der Außen- und Wirtschaftspolitik unterdrücken läßt. Die betonte Verneinung einer Wiedergutmachtungspflicht für die Ausbeutung in der Kolonialzeit, die starke Berücksichtigung von Eigeninteressen bei der Vergabe von Entwicklungshilfe und der Einbezug von außenpolitischen Uberlegungen in die Entwicklungshilfepolitik ${ }^{41}$ stoßen in der Bevölkerung of fensichtlich nur auf eine vergleichs-

38 J. von Stockhausen, Entwicklungshilfe im Zeichen von Geldknappheit und Pessimismus. "Entwicklung und Zusammenarbeit" Jg. 23 (1982), H. 4, S. 6.

39 Frankfurter Allgemeine Zeitung vom 14. 12. 1982.

40 Infratest Sozialforschung, Einstellung ... Materialband a.a.O. 1984, S. $134 \mathrm{ff}$.

41 Entwicklungspolitik, Hilfe ohne schlechtes Gewissen. "Rheinischer Merkur" vom 3. 8. 1984; abgedruckt in BMZ, Entwicklungspolitik - Spiegel der Presse 16/84, S. 453. 
weise geringe Resonanz. Wenn von der politischen Opposition die Betonung dieser außen- und wirtschaftspolitischen Rationalitätskalküle kritisiert wird, so kann sie sich einer breiten Zustimmung der deutschen Wahlbevölkerung gewiß sein. Entwicklungshilfepolitik ist - und diese Auffassung wird seit Beginn der deutschen Entwicklungshilfe immer wieder mit Nachdruck vertreten ${ }^{42}$ - ein untaugliches Instrument für die Verfolgung von kurzfristigen außenpolitischen und binnenwirtschaftlichen Interessen, auch wenn die Versuchung groß ist, sie für diese Bereiche einzusetzen, nicht zuletzt um Wählerstimmen in den begünstigten Bevölkerungsgruppen zu mobilisieren.

Eine solche politische Verengung der Entwicklungshilfe findet weder eine breite Zustimmung der deutschen Bevölkerung, noch wird sie den Schuldendienstproblemen gerecht, vor denen die Entwicklungsländer stehen.

\section{Von der Projekthilfe zur makroökonomischen Managementhilfe}

In jüngerer Zeit ist das offiziell proklamierte Anliegen der Entwicklungshilfe, durch Hilfe zur Selbsthilfe den "dynamischen Kräften" in den Entwicklungsländern die für ihre Entfaltung nötigen Werkzeuge an die Hand zu geben, unter verstärkte Kritik geraten. Dabei wird einmal auf die kontraproduktiven Wirkungen von Entwicklungshilfegeldern, zum anderen auf die Überbetonung von ökonomischen Entwicklungsengpässen hingewiesen.

Ausgangsüberlegung für die Kritik an den kontraproduktiven Auswirkungen von Entwicklungshilfegeldern ist der Umstand, daß etwa zwei Drittel der gesamten Entwicklungshilfe über die Regierungen der Entwicklungsländer geleitet werden ${ }^{43}$ mit der Folge, daß die Auswirkungen von Entwicklungshilfegeldern auf das gesamtwirtschaftliche Investitionsniveau von der staatlichen Budgetplanung abhängen. Dabei werden besonders kritisch die folgenden Möglichkeiten ihrer Beeinflussung durch Entwicklungshilfegelder herausgestellt:

- Umlenkung der eigenen Mittel des Empfängerlandes von investiven zu konsumtiven Ausgaben; als bezeichnen $\overline{d e}$ Indizien für eine solche Mittelumlenkung werden genannt: die steigenden Militärausgaben, die hohen Personalkosten in der öffentlichen Verwaltung, die wachsenden Refinanzierungsbedürfnisse zur Bedienung der Zinsund Tilgungsverpflichtungen ausländischer Darlehen.

- Substitution von Krediten zu Marktkonditionen und ein damit einhergehender nachlassender Druck, die Entwicklungshilfegelder auf die ertragreichsten Verwendungszwecke zu konzentrieren: so trägt nach Auffassung des Wissenschaftlichen Beirats beim BMZ die Bereitstellung weiterer Finanzmittel zu günstigen Bedingungen nicht dazu bei, die gegenwärtigen finanziellen Probleme langfristig zu lösen. Vielmehr füh-

42 Vgl. H. Ziock (Hrsg.), Entwicklungshilfe - Baustein für die Welt von morgen. Frankfurt/M. - Berlin 1966.

43 H. Sperber, The Efficiency Reducing Effects of Official Development Aid. "Intereconomics" March/April 1983, S. 85. 
re eine solche Therapie lediglich zu einer Verlagerung der Probleme in eine fernere Zukunft, wobei die sich dann ergebenden Anpassungslasten zwangsläufig größer sein würden als heute. ${ }^{44}$

- Substitutionen von Steuermitteln: Motiv für ein solches Verhalten ist das Bemühen zahlreicher Regierungen, die politische Gunst der Wachstumsbereiche zu erhalten bzw. zu gewinnen, die im gegenwärtigen Entwicklungsstadium die hauptsächlichen Steuersubjekte darstellen: gepaart hiermit führt das Bemühen zum Ausgleich der Leistungsbilanz dazu, die Entwicklungshilfemittel bevorzugt in die Wachstumsbereiche zu lenken mit der Folge, daß die Mittel nicht ausreichen, auch die Elendsbereiche zu beseitigen.

Das Verteilungsdilemma glaubte man anfänglich mit dem sogenannten "trickle down"Effekt überspielen zu können. Dieser Theorie zufolge würde der Wohlstand, den man anfänglich einer kleinen Schicht an der Spitze schafft, allmählich zu den übrigen sozialen Gruppen durchsickern. Diese Theorie hat sich aber nach weitverbreiteter Auffassung als falsch erwiesen.

Angesichts dieser Erkenntnis setzt sich in einem zunehmenden Maße die Auffassung durch, daß in der wirtschaftswissenschaftlichen Diskussion der Entwicklungshilfepolitik wohl zu lange die sozialen und politischen Aspekte der Entwicklung vernachlässigt worden sind. Nach Auffassung von Glismann et al. ist dieses darauf zurückzuführen, daß die Ókonomen in den entwickelten westlichen Ländern bei ihren Ưberlegungen in der Regel von Nationen als politische, soziale, kulturelle und ökonomische Einheiten ausgegangen sind. Dabei hätte man aber außer acht gelassen, daß in den meisten Entwicklungsländern die soziale und politische Integration, die in den alten Nationalstaaten erreicht worden ist, erst noch geschaffen werden muß. Mit anderen Worten, die Fehleinschätzung der Entwicklungshilfepolitik bestand lange Zeit darin, daß man glaubte, bei den Entwicklungsländern von funktionsfähigen nationalen Einheiten ausgehen zu können. Nur unter dieser Annahme konnte man die Förderung der wirtschaftlichen Entwicklung als die entscheidende strategische Variable zur Lösung des Entwicklungsproblems ansehen. ${ }^{45}$

Es sind die sozialen und politischen Engpaßbereiche, auf die sich Myrdal und Seers beziehen, wenn sie die vereinfachende Schlußfolgerung des Zweiten Brandt-Berichts ${ }^{46}$ kritisieren, daß die Massenarmut in den Entwicklungsländern automatisch einen Ressourcentransfer an ihre Regierungen rechtfertige; eine solche Politik spare die interne Politik der Hilfeempfänger aus: "in many countries the problem precisely is the government. It would be strange if providing it with money was any help to the poorest classes - any more than payments to the Mafia would necessarily help the peasants of Sicily ${ }^{47}$ Es

44 Wissenschaftlicher Beirat beim BMZ, Stellungnahme zu den Vorschlägen des Zweiten Brandt-Berichts. BMZ, Entwicklungspolitik - BMZ - aktuell, April 1983.

45 H. H. Glismann et al., Weltwirtschaftslehre. München 1982, S. 141 f.

46 W. Brandt (Hrsg.), Hilfe in der Weltkrise. Ein Sofortprogramm. Der 2. Bericht der Nord-Süd-Kommission. Reinbek 1983.

47 G. Myrdal, D. Seers, The bucks stop here. "The Guardian" vom 2. 2. 1982; abgedruckt in BMZ, Entwicklungspolitik - Spiegel der Presse 14/82, S. 422. 
müsse eine Unterscheidung gemacht werden zwischen Hilfe an die Wirtschaft und Hilfe für die Armen. In gleicher Weise haben sich $\mathrm{Lal}^{48}$ sowie Bauer und Yamey ${ }^{49}$ geäußert. Aus dieser Einschätzung heraus wird als einzige Hilfeart, die in einigen Fällen die Bezeichnung Hilfe verdient, diejenige bezeichnet, von der der Hilfegeber absolut sicher sein kann, daß sie für die Befriedigung von Grundbedürfnissen, wie sauberes Wasser oder Gesundheitsvorsorge verwandt wird. Eine solche Hilfeform sei die Katastrophenhilfe. ${ }^{50}$ Eine andere Auffassung wird von denjenigen vertreten, die dafür plädieren, die identifizierte soziale und politische Lücke verstärkt zum Gegenstand der Entwicklungshilfepolitik zu machen. Nach über mehr als 20 Jahren zum Teil schmerzlicher Erfahrung mit den Problemen der Entwicklung der Dritten Welt sollte es nach Lösch allmählich deutlich geworden sein, daß im Konzept unserer sogenannten "Entwicklungszusammenarbeit" eine wesentliche Komponente fehlt, und zwar die des Sozialtechnologie-Transfers in die Entwicklungsländer, schlagwortartig vereinfacht, die Ubertragung von ordnungs- und wirtschaftspolitischem Know-how. ${ }^{51}$ Der Transfer von technischem und betriebswirtschaftlichem Managementwissen müsse ergänzt werden durch einen verstärkten Transfer der in den Industrieländern vorhandenen Kenntnisse darüber, wie eine Volkswirtschaft zu organisieren und zu "managen" sei. Wenn dieser Transfer bis heute nicht den ihm im Rahmen der westlichen Entwicklungshilfe zukommenden Rang einnimmt, so sei dieses offenbar die Folge der übergroßen Empfindlichkeit der meisten Entwicklungsländer in allen Fragen, die irgendwie ihre nationale Souveränität tangieren, und der Furcht der Geberländer, an solchen Empfindlichkeiten zu rühren. "Aus dieser Furcht heraus nehmen sie of fenbar lieber die durchaus reale Gefahr der weitgehenden Nutzlosigkeit ihrer Kapital- und Technischen Hilfe in Kauf, als deren Vergabe von bestimmten Wirtschaftssystem- oder Wirtschaftspolitikkriterien abhängig zu machen"..52

Lösch spricht hier einen Bereich der Entwicklungshilfepolitik an, der zwar, wie das Soforthilfeprogramm der Weltbank deutlich macht, eine besondere Aktualität hat, dessen Bedeutung aber bereits in den sechziger Jahren sehr wohl erkannt worden ist. So weist Rau mit Nachdruck darauf hin, daß ein Transfer von Kapital und Fachkräften allein nicht genügt, um automatisch einen ökonomischen Entwicklungsprozeß in Gang zu bringen. "Wenn Entwicklungsförderung als igelenkter Kulturwandel verstanden wird, so bleibt nur Raum für eine aufgrund sorgfältiger Analyse ermittelte Rezeptur von Maßnahmen und Maßnahmenkonditionen, die an allen denkbaren und möglichen Punkten

48 D. Lal, The Poverty of 'Development Economics'. London 1983, S. 55 f.

49 P. Bauer, B. Yamey, Why we should close our purse to the Third World. "The Times" vom 11.4. 1983; abgedruckt in BMZ, Entwicklungspolitik - Spiegel der Presse 8/83, S. 231.

50 G. Myrdal, D. Seers, a.a.O. 1982, S. 422 - J. Galtung, N o country has developed by borrowing money. "The Guardian" vom 25. 2. 1983; abgedruckt in BMZ, Entwicklungspolitik - Spiegel der Presse 5/83, S. 144.

51 D. Lösch, Markt oder Staat für die Dritte Welt? Wirtschaftssystem und Wirtschaftspolitik in Entwicklungsländern, illustriert am Beispiel der Republik Malawi. Hamburg 1983, S. 18.

52 D. Lösch, a.a.O. 1983, S. 20. - Im gleichen Sinne hat Pieroth jüngst die Projekthilfe kritisiert, wenn e r darauf hinweist, daß der Nachteil der Fixierung auf Einzelprojekte darin bestehe, daß dabei deren Umwelt, d. h. die wirtschaftliche und politische Ordnung, in der Projekte unterstützt werden, vernachlässigt werde. E. Pieroth, Die Ordnung bestimmt die Entwicklung. "Wirtschaftswoche" Nr. 39 vom 21. 9. 1984; abgedruckt in BMZ, Entwicklungspolitik - Spiegel der Presse 20/84, S. 590. 
der ökonomischen, sozialen und gesellschaftlichen Umwelt angesetzt werden, die von außen her überhaupt zugänglich sind. "53 $^{53}$

Zwei Aspekte der Überlegungen von Rau verdienen eine besondere Beachtung. Zunächst ist Entwicklungsförderung "gelenkter Kulturwandel". Dieses bedeutet, daß Entwicklungsförderung niemals unpolitisch ist, mit anderen Worten, daß Entwicklungsförderung nolens volens eine Einmischung in die inneren Angelegenheiten des jeweiligen Empfängerlandes ist. Darüber hinaus verliert Entwicklungsförderung in dem Umfange an Effizienz, wie ihr die zu erreichenden Ansätze der ökonomischen, sozialen und gesellschaftlichen Umwelt nicht zugänglich sind. Rau spricht damit aus zwei Blickwinkeln die Souveränität der Empfängerländer an.

Es war das Bemühen, die Souveränität der jungen Staaten zumindest verfahrensmäßig zu respektieren, das die Entwicklungshilfegeber dazu veranlaßte, der Entwicklungshilfe das Antragsprinzip zugrunde zu legen, demzufolge die Initiative der Zusammenarbeit von den Entwicklungsländern ausgehen soll. Das Antragsprinzip mag zwar außenpolitische Früchte, zumindest kurzfristig, gebracht haben, entwicklungshilfepolitisch hat es die Geberländer und hier insbesondere die Entwicklungshilfe-Administration an die kurze Leine gelegt, und zwar in dem Sinne, daß entwicklungsstrategische Betrachtungen zugunsten tagespolitischer Erwägungen das Nachsehen hatten. ${ }^{54}$

Nach Auffassung von Entwicklungshilfeminister Dr. Warnke hat die Bundesregierung in der Vergangenheit all zu oft nur defensiv auf Forderungen und Wünsche der Dritten Welt reagiert; es sei an der Zeit, besser als bisher auch eigene Vorstellungen in die partnerschaftliche Zusammenarbeit mit den Entwicklungsländern einzubringen. Den Kern des von Warnke angesprochenen Dialogs hat Liefer bereits vor nahezu 20 Jahren in der Weise formuliert, daß es entscheidend darauf ankomme, in welchem Geist gegeben und in welchem Geist Entwicklungshilfe empfangen werde. "Hier ist der geistige Ausgangspunkt, um uns durchzuringen zu einer Partnerschaft, die am besten die Dinge zwischen den Menschen regelt. "\$s Eine UUberbetonung der eigenen Interessen wirtschaftlicher oder/und außenpolitischer Art kann sich leicht in das Gegenteil von dem verkehren, was das eigentliche Eigeninteresse der Entwicklungshilfepolitik ist, nämlich über die Schaffung von Massenkaufkraft Märkte von morgen aufzubauen und zur Vorbeugung von politischen Krisen die Verteilung der Wirtschaftsgüter zwischen Armen und Reichen stärker auszugleichen. Um den Sozialtechnologietransfer zu einem wirksamen Instrument der deutschen Entwicklungshilfepolitik zu machen, ist ein Klima des Politikdialogs erforderlich, das den Regierungen und der Bevölkerung in den Entwicklungsländern Vertrauen gibt, von dem Angebot dieser Hilfe Gebrauch zu machen. Eine solche Form der bilateralen Hilfe kann und darf in ihrem eigenen Interesse nicht vorrangig darauf angelegt sein, kurzfristig "politischen 'Good-willı zu kassieren ". ${ }^{56}$

$53 W$. Rau, Der Frieden ist unteilbar. In: H. Ziock, a.a.O. 1966, S. 40.

54 J. von Stockhausen, Auf aktiver Projektsuche in der Dritten Welt. Vom Antrags- zum Angebotsprinzip in der Entwicklungshilfe. "Frankfurter Allgemeine Zeitung" vom 20.4. 1983; abgedruckt in BMZ, Entwicklungspolitik - Spiegel der Presse 9/83, S. 262 f.

$55 W$. Liefer, Es genügt nicht, einfach das Scheckbuch zu ziehen ... In: H. Ziock, a.a.O. 1966, S. 155.

56 F. K. Vialon, Motive deutscher Entwicklungspolitik. In: H. Ziock, a.a.O. 1966, S. 44. 


\section{Wider das Sankt Florians Prinzip in der Entwicklungshilfe}

Die Zahl der Entwicklungshilfegeber ist groß. Es wird berichtet, daß beispielsweise in dem kleinen Land Obervolta mit 6,3 Mio. Einwohnern mehr als 300 Entwicklungsorganisationen tätig sind. Soll man über eine so vielfältige Hilfsbereitschaft froh oder entsetzt sein? Es sind zwei Gesichtspunkte, die es bei dieser Frage zu berücksichtigen gilt.

Wie bereits erwähnt, kann der Zufluß von öffentlicher Entwicklungshilfe bewirken, daß ein Teil der im Budget der Empfängerland-Regierung eingeplanten oder bereits vorhandenen Finanzmittel von den Verwendungszwecken, für die öffentliche Entwicklungshilfe gewährt wird, befreit und für andere Verwendungszwecke eingesetzt wird. Eine solche Freisetzung von Budgetmitteln ist von der Empfängerland-Regierung um so leichter durchzusetzen, je größer die Zahl der Entwicklungshilfe-Anbieter ist und je geringer die Koordination bzw. die entwicklungsstrategische Absprache zwischen den Geberländern ist. "Durch 'Hausieren ' unter den Geberländern und eine Politik des 'Moral Hazard haben die Entwicklungsland-Regierungen gute Chancen, genau jene oder ähnliche Projekte als finanzierungswürdig vorzuschlagen und durchzusetzen, die sie auch ohne Entwicklungshilfe hätten realisieren können ". ${ }^{57}$

Kompensatorische Budgetreaktionen lassen sich durch die Regierungen der Entwicklungsländer um so leichter durchführen, je unterschiedlicher die Motivstruktur für die Vergabe von Entwicklungshilfe ist. Eine Motivanalyse der wichtigsten Geberländer macht gewichtige Unterschiede deutlich. Die Entwicklungshilfe der USA ist stark militärstrategisch orientiert, für Großbritannien und Frankreich stehen die ehemaligen Kolonien politisch im Vordergrund. Dabei ist es ein wesentliches Ziel der französischen Leistungen, die politische Ausstrahlung Frankreichs und die Bedeutung der französischen Sprache und Kultur in der ehemaligen Communauté zu erhalten. Für Japan stand von Anfang an der Gedanke der Exportförderung im Vordergrund. ${ }^{58}$

Und wie steht es um die Motive der deutschen Entwicklungshilfe? Ende der sechziger bis Anfang der siebziger Jahre hatten sich Bemühungen durchgesetzt, Entwicklungshilfepolitik als eigenständige Aufgabe zu verstehen, die nicht mit der bundesrepublikanischen Außen- und Wirtschaftspolitik identisch ist. ${ }^{59}$ Die politische Eigenständigkeit der Entwicklungshilfe ist gefährdet, oder anders ausgedrückt, die regierungsamtlichen Motive der deutschen Entwicklungshilfe nähern sich denen Japans an, wenn nach Auffassung von Entwicklungshilfeminister Dr. Warnke die Entwicklungshilfe stärker mit den wirtschaftlichen Interessen der Bundesrepublik verknüpft werden müsse: "Wir suchen Projekte aus, wo deutsche Lieferungen wettbewerbsfähig sind, wo das Geld des Steuerzahlers vernünftig angelegt ist. "60

57 H. Sperber, Erfolgsbedingungen der Offentlichen Entwicklungshilfe. Schriften zur Monetären Okonomie Bd. 21, Baden-Baden 1984, S. 51.

58 Chr. Uhlig, Entwicklungshilfepolitik. Hamburg 1971, S. $240 \mathrm{ff}$.

59 Chr. Uhlig, a.a.O. 1971, S. 48.

60 NRZ-Gespräch mit dem Entwicklungsminister Warnke: Unverkrampft und ohne Ideologie. "Neue RuhrZeitung" vom 31. 8. 1984; abgedruckt in BMZ, Entwicklungspolitik - Spiegel der Presse 18/84, S. 514. 
Es sind nicht so sehr die Kosten, die den Entwicklungsländern durch eine solche Ausrichtung entstehen und die den Netto-Effekt der Entwicklungshilfe schmälern, ${ }^{61}$ als vielmehr die relative Bedeutung von christlich-humanitären Motiven im Rahmen des Motivbündels bei der Vergabe von öfentlicher Entwicklungshilfe, die an Gewicht zu verlieren droht. Entwicklungshilfepolitik, die sich auch oder sogar vor allem der sozialen Frage dieses Jahrhunderts stellt, ${ }^{62}$ greift zu kurz, wenn sie ihre Aufgabe auf einen Ressourcentransfer in die armen Länder der Dritten Welt verkürzt. Sie bedient sich des Sankt Florians Prinzips, wenn die starke Vertretung von wirtschaftlichen Eigeninteressen mit dem Hinweis zu rechtfertigen versucht wird, daß wir uns "von Amerikanern, Japanern, Franzosen nicht die Butter vom Brot nehmen lassen" wollen. ${ }^{63}$

Unter Bezugnahme auf die laufende Diskussion über die Auswirkungen der Agrarexporte von Entwicklungsländern spricht Schmitt den zentralen Aspekt einer christlich-humanitären Entwicklungshilfe an, wenn er die Bedeutung von umfassenden Reformen der politischen Verfassung, die zur Verwirklichung demokratischer und freiheitlicher Grundrechte in den Entwicklungsländern führen, herausstellt. Der politische Willensbildungs- und Entscheidungsprozeß könne wohl nur dann unsere Zustimmung finden, wenn er die Verwirklichung der demokratischen Grundrechte ermöglicht. ${ }^{64}$ In diesem Sinne gehört zu den neuen Akzenten der deutschen Entwicklungshilfepolitik der Versuch, die Partner in der Dritten Welt davon zu überzeugen, daß es darauf ankommt, marktwirtschaftliche Elemente und pluralistische Strukturen in Wirtschaft und Gesellschaft zu fördern; denn nur so werde eine Entfaltung der schöpferischen Kräfte breiter Bevölkerungsschichten ermöglicht, auf die es heute mehr denn je ankomme. ${ }^{65}$

Was hier durch bilaterale Zusammenarbeit angestrebt wird, ist konzeptionell das Pendant zu der schon seit längerem proklamierten Grundbedürfnisstrategie, deren essentielles Wesensmerkmal eine stärkere Beteiligung der Betroffenen bei der Planung und Durchführung von Entwicklungsmaßnahmen im Sinne einer selbstbestimmten Entwicklung ist. Es sind im wesentlichen zwei Fragen, die sich aufdrängen, wenn die Entwicklungshilfepolitik stärker auf eine Änderung der wirtschaftlichen und politischen Ordnung in den Entwicklungsländern ausgerichtet werden soll.

Das bisher angewandte Antragsprinzip der öffentlichen Entwicklungshilfe hat zur Folge, daß nur die Regierungen der Entwicklungsländer Zugang zur öffentlichen Entwicklungshilfe haben. Privaten gesellschaftspolitischen Gruppierungen ist der Zugang verwehrt, sofern er von den jeweiligen Regierungen nicht gewünscht wird. An dieser verfah-

$61 S$. Schulz et al., Tendenzen der Entwicklungspolitik der westlichen Industrieländer. Forschungsauftrag des Bundesministeriums für wirtschaftliche Zusammenarbeit. Bonn 1981, S. 36 ff.

62 Kirchenamt im Auftrage des Rates der Evangelischen Kirche in Deutschland (Hrsg.): Landwirtschaft im Spannungsfeld zwischen Wachsen und Weichen, Okologie und Okonomie, Hunger und Uberfluß. Eine Denkschrift der Kammer der Evangelischen Kirche in Deutschland für soziale Ordnung. Gütersloh 1984, S. 16.

63 NRZ-Gespräch, a.a.O. 1984, S. 514.

64 G. Schmitt, Anmerkungen zu einer Denkschrift der Evangelischen Kirche zur Agrarpolitik. "Agrarwirtschaft" Jg. 33 (1984), H. 7, S. 220.

65 BMZ, Deutsche Entwicklungspolitik a.a.O. 1984, S. 4. 
rensmäßigen Schwäche ändert auch wenig die von der Bundesregierung betonte Bedeutung der nichtstaatlichen Zusammenarbeit, die darauf ausgerichtet sei, die hohe Mobilisierungskraft ihrer Zielgruppen in den Entwicklungsländern zu nutzen. ${ }^{66}$ Darüber hinaus beinhaltet das Antragsprinzip die Gefahr, daß die Empfänger ein bestimmtes Volumen an Entwicklungshilfe gleichsam als Rechtsanspruch ansehen, wodurch, nicht zuletzt aus außenpolitischen Gründen, die mögliche Flexibilität des Instruments Entwicklungshilfe zur Uberzeugung der Empfängerland-Regierungen für ordnungspolitische Reformen eingeschränkt wird. ${ }^{67}$

Nach Auffassung der Bundesregierung haben einige Länder bereits große Anstrengungen zur Korrektur ihres wirtschafts- und sozialpolitischen Kurses unternommen und dabei ihrer Bevölkerung zum Teil große Opfer auferlegt. ${ }^{68}$ Die Frage ist, ob solche Opfer durch die herkömmliche Projekthilfe aufgefangen werden können oder ob hier nicht eine stärker zielorientierte Ausgestaltung der Entwicklungshilfe in Form von "Entwicklungspolitik-Krediten" am Platze ist, wie sie z. Z. in der Weltbank diskutiert werden. Eine solche Umorientierung hätte zur Folge, daß der Dialog über makroökonomische Reformen nicht mit projektspezifischen Erfordernissen vermengt und dabei in beider Hinsicht "verdünnt" würde. Darüber hinaus würde auf diese Weise die Verantwortlichkeit für projektspezifische Erfolgsbedingungen stärker in die Verantwortlichkeit der Empfängerländer verlagert.

Pieroth spricht insbesondere die Verfahrensregeln des Politikdialogs an, wenn nach seiner Auffassung mit Ländern, deren Politik von freier Initiative und Marktorientierung geleitet wird, die of $t$ mit partnerschaftlicher Würde kaum zu vereinbarenden Detaileingriffe und -bedingungen unserer Projektförderung großzügiger, bis hin zur Budgethilfe, gehandhabt werden können. ${ }^{69}$ Die Bundesregierung hält zwar nach wie vor an dem Grundsatz fest, ihre Zusagen für bestimmte Projekte und Programme zu geben und keine Budget- oder Zahlungsbilanzhilfe zu leisten, ${ }^{70}$ zugleich hat sie aber die Führungsrolle von multilateralen Institutionen bei dem Dialog über makroökonomische Fragen anerkannt. ${ }^{71}$ Letzteres darf und kann aber nicht heißen, die Suche nach geeigneten Lösungen für die Problematik des Zusammenhanges von Verschuldung und Entwicklungshilfe allein den multilateralen Institutionen, wie etwa Weltbank und Internationaler Währungsfonds, zu überlassen. Unorthodoxe Vorschläge, wie etwa alte Schulden durch Entwicklungshilfe abzugelten, um den Entwicklungsländern die Möglichkeit zu geben, die gesparten Summen im Inland für eine Entwicklung aus eigener Kraft und frei von Export-

66 BMZ, Deutsche Entwicklungspolitik a.a.O. 1984, S. 4.

$67 \mathrm{~J}$. Betz, Die Internationalisierung der Entwicklungshilfe. Aachener Studien zur internationalen technischwirtschaftlichen Zusammenarbeit Nr. 19. Baden-Baden 1978, S. $189 \mathrm{ff}$.

68 BMZ, Deutsche Entwicklungspolitik a.a.O. 1984, S. 4.

69 E. Pieroth, a.a.O. 1984, S. 590.

70 BMZ, Deutsche Entwicklungspolitik a.a.O. 1984, S. 23.

71 BMZ, Deutsche Entwicklungspolitik im Jahre 1982. Memorandum der Bundesregierung zur DAC-Jahresprüfung 1983/84. Entwicklungspolitik - BMZ - aktuell August 1983, S. 18. 
zwängen einzusetzen, ${ }^{72}$ oder bei Umschuldungsverhandlungen die Finanzierung von Landreformprogrammen, von Projekten zur Erstellung von vorrangig arbeitsintensiven Massenkonsumgütern ${ }^{73}$ etc. anzubieten, sollten stärker aufgegriffen und in die internationale Diskussion eingebracht werden.

Die Verschuldungslage der Entwicklungsländer und ihre Ursachen machen die Suche nach neuen Wegen der Entwicklungshilfepolitik erforderlich. Das Aufspüren von solchen neuen Wegen markiert ihre Möglichkeiten und Grenzen in der Zukunft.

72 IWF hegt trügerische Hof f nungen. "Frankfurter Rundschau" vom 21. 9. 1984; abgedruckt in BMZ, Entwicklungspolitik - Spiegel der Presse 20/84, S. 585.

73 H. Elsenhans, a.a.O. 1984, S. 129 f. 


\section{ABSTRACTS}

\section{Peace and War in International Law}

\section{By Wolfgang Graf Vitzthum}

War as an elemental form of conflict has always been a central concern of international law, with particular references to questions of formal and material states of "peace" and the right to wage war as well as the duties of belligerents.

From Roman times until the modern era a trend can be discerned from definitions of rightful recourse to armed force towards a prohibition of the use of force, reflecting the dramatic increase of the destructive power of military weapons.

The UN-based for safeguarding peace, while resting on the general proposition of prohibiting the use of armed force, has been impeded in its efficacy by the conflicting interests of the member states enjoying veto rights in the Security Council, the tendency towards employment of UN forces for surveillance rather than enforcement action and the fluid relationship between the right of self-defence and interventionist ambitions of states.

The advent on the stage of international politics of new states, as successors to former colonies, has accentuated the problem of acceptance of a historical international legal order by those who had no opportunity to influence its creation. Peaceful change and appropriately equitable international procedures have therefore acquired added significance as elements of legal safeguards against military conflict. The international legal norms governing the conduct of war remain indispensable in this context even though they are not a means of avoiding war.

International law can thus serve as important catalyst in the efforts to reconcile the demands for both non-violence and justice in a nuclear age.

\section{The Indebtedness of Developing Countries and its Consequences for Official Development} Aid

\section{By Joachim von Stockhausen}

The increasing indebtedness of developing countries has called into question several aspects of the conventional development aid approach. The World Bank has begun to complement traditional project aid by granting sector loans to support borrower countries more efficiently. Other donors have been requested to consider similar actions.

The development aid policy of the German Federal Government is part of its external relations with the countries of the Third World. An official shift from altruistic to more 
self-interested motives is one of the reasons for a widening gap between the German public's practical formulation of government policy.

The officially proclaimed goal of strengthening the dynamic forces in the developing countries by giving aid for supporting self-help efforts is criticized for two reasons: first, about two thirds of of ficial development aid is given to the governments of developing countries, and it is their behaviour which strongly influences the developmental effects of the aid; second, economic development contraints have been overstated at the expense of socio-political constraints. This view has led to two proposals for future aid strategy: one is to give aid only where the donor is absolutely certain that it will be used for elementary needs in a really poor country; the other is to strengthen so-called "social-technologytransfer". The latter strategy demands a climate of policy dialogue which gives confidence to the governments and the populations of developing countries to make use of aid. A development aid policy which is dominated by shortterm political and economic self-interest tends to be counter-productive to such a policy dialogue and prevents efforts to look for new solutions to overcome the present development crisis of the countries of the Third World.

\section{South Africa - Prepotency and Impotence}

\section{By Klaus Frhr. von der Ropp}

As is well known, the administration of former U.S. president Jimmy Carter was deeply involved in trying to overcome Apartheid; it naively assumed that it could help to introduce the "American model" at the Cape of Good Hope. The Reagan administration, on the other hand, is prepared to admit that Apartheid, because of South Africa's uniquely heterogeneous population, must be replaced by a new order sui generis. Both administrations failed, as Pretoria is not prepared to negotiate with the true representatives of Black South Africa.

South Africa dominates developments in most parts of Southern Africa more or less with ease. But Pretoria is totally unable to solve its domestic problems. Consequently there have been, since August 1984, more serious uprisings in many parts of the country than ever before. There is open anarchy in many Black townships. In many of them the often very young revolutionaries have succeeded in making them ungovernable. Even if many leading white South Africans today realise that Apartheid was an error, they do not know by which new order to replace it. The future holds for South Africa a very bloody civil war. This is clearly recognised by Western and other economic interest groups; they realise that for a long time South Africa will have lost its attractiveness as an economic partner. Today American Republicans impose sanctions against South Africa that American Democrats had hardly thought of only a few years ago. But it still holds true that any solution which is to bring liberation to South Africa must include copper-bottomed guarantees of secure existence for Whites. 